\title{
Theragnostic Magnetic Core-Shell Nanoparticle as Versatile Nanoplatform for Magnetic Resonance Imaging and Drug Delivery
}

\author{
Samira Zarei $^{2}{ }^{\mathbb{D}}$, Somayeh Sadighian ${ }^{1 \mathbb{D}}$, Kobra Rostamizadeh ${ }^{3, *(\mathbb{D})}$, Maryam Khalkhali ${ }^{4} \mathbb{( \mathbb { D }}$

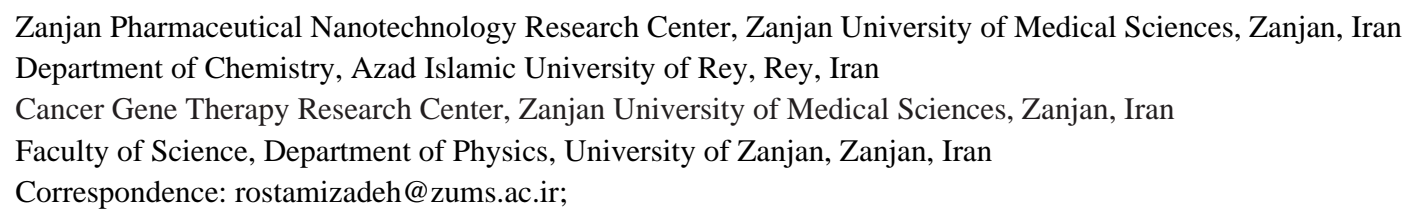

Received: 7.12.2020; Revised: 30.01.2021; Accepted: 2.02.2021; Published: 8.02.2021

\begin{abstract}
In this study, magnetic core-shell (MCS) nanoparticles were prepared as theragnostic potential nanoplatforms for simultaneously targeted drug delivery systems for tamoxifen and diagnosis. MCS nanoparticles were prepared in a well-shaped spherical form by the o/w emulsion method and characterized by means of dynamic light scattering (DLS), Scanning electron microscopy (SEM), Nuclear magnetic resonance (NMR), transform infrared (FT-IR) spectroscopy, and vibrating sample magnetometer (VSM). Scanning electron microscopy (SEM) indicated spherical nanostructures' formation with the final average particle size of around $80 \mathrm{~nm}$. The findings proved the superparamagnetic properties of the MCS nanoparticles with relatively high-magnetization values $(11.69 \mathrm{emu} / \mathrm{g})$, which indicate that they were sensitive enough to external magnetic fields as a magnetic drug carrier. The nanoparticles showed $8.14 \%$ and $52.19 \%$ drug loading and encapsulation efficiency, respectively. MCS nanoparticles showed sustained release behavior for $120 \mathrm{~h}$ in the phosphate-buffered saline (PBS, $\mathrm{pH}=7.4,5.4)$ at $37{ }^{\circ} \mathrm{C}$. The ratio between transverse and longitudinal relaxivity $(\mathrm{r} 2 / \mathrm{r} 1)$ value of the MCS nanoparticles was around 20, indicating their potential as a T2 MRI contrast agent. It can be concluded that the prepared MCS nanoparticles may serve as a promising carrier as an MRI contrast agent and targeted controlled anticancer drug delivery.
\end{abstract}

Keywords: theragnostic; mPEG-PCL; tamoxifen; magnetite; contrast agent; controlled release.

(C) 2021 by the authors. This article is an open-access article distributed under the terms and conditions of the Creative Commons Attribution (CC BY) license (https://creativecommons.org/licenses/by/4.0/).

\section{Introduction}

The term "theranostics" defines a new methodology to combine the modalities of the therapeutic and diagnostic. The most attractive feature of the theragnostic concept is to develop specific and individualized therapeutic strategies for personalized medicine. Combining therapeutic and diagnostic capabilities into one single agent can tailor a treatment protocol according to the test results, consequently tuning more specific, personalized, and efficient protocols for treatment. Magnetic resonance imaging (MRI) is a powerful nonaggressive imaging method with very high resolution giving a chance for careful determination of the 3D figure to distinguish soft body tissue [1]. However, precise diagnosis and essential contrast between normal tissues and abnormal tissues need contrast agents. Magnetic nanoparticles (MNPs) are a negative contrast agent that affects the transverse $\left(\mathrm{T}_{2}\right)$ relaxation time and cause a darker state in the $\mathrm{T}_{2}$-weighted image that was gathered in tissue. Also, MNPs, due to their 
superparamagnetic property, possess the potential for being guided through an external magnetic field and act as a targeting moiety for different drug delivery systems [2, 3]. In contrast to the high potential of MNPs as imaging, diagnostic and targeting agent for the preparation of various theragnostic, they suffer from a high tendency to agglomeration, low loading capacity, and short half-life in body fluids as a drug delivery system. To overcome these shortcomings, MNPs nanostructures with different polymer shells have been introduced [4-8]. In these structures, MNPs act as a targeting potential and imaging agent, while polymers prevent their aggregation and simultaneously increase loading capacity and circulation time. Xie et al. [9] have investigated the in vivo MRI contrast properties of polyethylene glycol (PEG), PEG/PEI (polyethyleneimine), and PEG/PEI/Tween 80 modified superparamagnetic iron oxide nanoparticles (SPION). They showed various vascular imaging effects after $24 \mathrm{~h}$ intravenous injections of the prepared samples. Yoon et al. [10] developed a theragnostic agent composed of epirubicin (EPI) loaded ultra-small superparamagnetic $\mathrm{Fe}_{3} \mathrm{O}_{4}$ nanoparticle and poly aspartic acid, which showed a high relaxivity, $\mathrm{r}_{2}$ value, and cytotoxic against cancer cells.

Poly ( $\varepsilon$-caprolactone)/poly(ethylene glycol) (mPEG-PCL) copolymers because of biodegradable, biocompatible, and amphiphilic properties are considered as a favorable candidate for the formulation of advanced drug carrier [11, 12]. PEG-PCL copolymers have attracted the most attention for poorly water-soluble drug entrapment and increased their water solubility and bioavailability due to PCL's hydrophobic segment. Besides, the hydrophilic PEG block can potentially enhance the circulation time of drugs. It can prohibit recognition by macrophages of the reticuloendothelial system (RES) after intravenous injection [13].

Tamoxifen is mostly used for post-menopausal women and prophylactic therapy. Due to important side effects such as drug resistance and endometrial cancer, tamoxifen demands targeted transfer to the tumor site and increased uptake by the tumor cells [14, 15].

This paper follows our previous study on developing MNPs coated with chitosan and folic acid-conjugated chitosan as a trimodally-targeted nanomagnetic on-theragnostic system [16]. We report the preparation of magnetic core-shell nanoparticles (MCS) as theragnostic nanoplatforms for tamoxifen delivery. Amphiphilic mPEG-PCL copolymers were used on the surface of MNPs, and MNPs play the role of contrast agent for imaging (MRI) when exposed to an alternating magnetic field.

\section{Materials and Methods}

\subsection{Materials.}

In this in-vitro study $\varepsilon$-caprolactone ( $\varepsilon$-CL) obtained from Sigma, stannous octoate ( $\mathrm{Sn}$ $(\mathrm{Oct})_{2}$ ) obtained from Acros. dicyclohexylcarbodiimide (DCC), poly (ethylene glycol) methyl ether (mPEG, $\mathrm{M}_{\mathrm{w}}=5 \mathrm{KDa}$ ), 4-dimethylamiopryidine (DMAP), polyvinyl alcohol (PVA, $\mathrm{M}_{\mathrm{w}}$ : 13-23 KDa), and chloroform, ferric chloride hexahydrate, ferrous chloride tetrahydrate, and $\mathrm{NH}_{4} \mathrm{OH}(25 \%)$ were from Merck. Dialysis tubing (molecular weight cutoff 12-14 KDa) were purchased from Sigma.

\subsection{Synthesis of $m P E G / P C L$ di-block copolymer.}

The preparation of the polymer is illustrated in Fig. 1. The mPEG-PCL was prepared in dry toluene by ring-opening polymerization of $\varepsilon$-caprolactone using $\mathrm{mPEG}$ as initiator and $\mathrm{Sn}(\mathrm{Oct})_{2}$ as a catalyst. Briefly, $\mathrm{mPEG}$ was added in $25 \mathrm{~mL}$ of dry toluene under nitrogen flow in a $150 \mathrm{~mL}$ dried round-bottom flask. Then, some drops of $\mathrm{Sn}(\mathrm{Oct})_{2}$ solution initiated the 
polymerization reaction, and the blend was refluxed under nitrogen for $18 \mathrm{~h}$ at $120{ }^{\circ} \mathrm{C}$ under magnetic stirring. Then cool to room temperature, the resultant mPEG-PCL copolymer was precipitated by quenching in cold ethyl ether. The product was dried in a vacuum oven at room temperature for $12 \mathrm{~h} \mathrm{[17].}$

\subsection{Synthesis of hydrophobic magnetite nanoparticles (MNPs).}

The hydrophobic magnetic nanoparticles were prepared by the co-precipitation technique from an aqueous $\mathrm{Fe}^{3+} / \mathrm{Fe}^{2+}$ solution (molar ratio 2:1). Briefly, a known quantity of $\mathrm{FeCl}_{3}$ and $\mathrm{FeCl}_{2}$ were dissolved in $50 \mathrm{ml}$ distilled water, and $3 \mathrm{~mL}$ of ammonium hydroxide (5 M) was added quickly. Then the oleic acid was added drop by drop and stirred [18]. The solution was collected by an external magnet and washed three times with distilled water and ethanol to remove excess oleic acid.

\subsection{Preparation of magnetic core-shell nanoparticles.}

Magnetic core-shell (MCS) nanoparticles were synthesized by the emulsion evaporation method (oil-in-water). Briefly, di-block copolymer (mPEG-PCL, $50 \mathrm{mg}$ ), oleic acid-coated magnetite $(5 \mathrm{mg})$, and tamoxifen $(10 \mathrm{mg})$ were dissolved in $10 \mathrm{~mL}$ of dichloromethane (DCM). The solution was added drop-wise into the aqueous PVA solution $(0.5 \% \mathrm{w} / \mathrm{v}, 50 \mathrm{~mL})$ under mechanical stirring (RW20, IKA, Germany) to gain an o/w emulsion. The organic solvent evaporated slowly to form the micelles. Nanoparticles were separated by centrifugation (14000 rpm) and washed with water several times, and finally freeze-dried at a pressure of $14 \mathrm{~Pa}$ and $78^{\circ} \mathrm{C}$ (EYELA, 2100, Tokyo, Japan).

\subsection{Characterization of the MPEG-PCL copolymers and drug-loaded MCS nanoparticles.}

\subsection{1. ${ }^{1} \mathrm{HNMR}$.}

The copolymer's chemical structure was recognized by nuclear magnetic resonance spectroscopy $\left({ }^{1} \mathrm{H} \mathrm{NMR}\right.$ ) in $\mathrm{CDCl}_{3}$ at $400 \mathrm{MHz}$ (Bruker, Advance 400). ${ }^{1} \mathrm{H}-\mathrm{NMR}$ spectra were obtained with $\mathrm{CDCl}_{3}$ as solvent and TMS as internal standard, using a Bruker $400 \mathrm{MHz}$ apparatus at $25^{\circ} \mathrm{C}$.

\subsubsection{FTIR analysis.}

To confirm the drug's presence in the nanoparticles and the interaction between the components, the end product spectrum and the initial materials were compared. FTIR spectra were recorded by $\mathrm{KBr}$ disks in the range of 400 to $4000 \mathrm{~cm}^{-1}$ [19].

\subsubsection{DSC analysis.}

Interaction between drug and copolymer or physical changes was studied using thermal analysis (DSC) (Mettler Toledo, model Star SW 9.30, Selangor, Switzerland). Samples were heated at a rate of $10{ }^{\circ} \mathrm{C} \mathrm{min}-1$, and the data were recorded from 0 to $200{ }^{\circ} \mathrm{C}$. (Mettler Toledo, model Star SW 9.30, Selangor, Switzerland). 
2.5.4. Scanning electron microscopy (SEM) and Transmission electron microscopy (TEM) analysis.

Philips CM100 electron microscope operating at $20 \mathrm{KV}$ and CM120 TEM (Philips) (accelerating voltage: $200 \mathrm{kV}$ ) were used to obtain SEM and TEM images of MCS nanoparticles.

\subsubsection{Size and zeta potential analysis.}

The size and zeta potential of the synthesized nanoparticle was determined by a Nano/zeta sizer (Malvern Instruments, Nano ZS, Worcestershire, UK) working on the dynamic light scattering (DLS) platform.

2.5.6. Vibrating sample magnetometer (VSM) analysis.

VSM (Lakeshore 7400, United States) was applied to investigate the magnetic properties of synthesized nanoparticle and hysteresis loops at room temperature from -20000 to 20000 Oe.

\subsubsection{Determination of drug loading and encapsulation efficiency.}

Briefly, $50 \mathrm{mg}$ MCS was added to $10 \mathrm{mg}$ of tamoxifen in $5 \mathrm{~mL}$ ethanol. Then the mixture stirred for $12 \mathrm{~h}$. The tamoxifen-loaded MCS were separated by an external magnet and then washed three times with deionized water. The tamoxifen entrapped nanoparticles were dried in a vacuum oven at $30^{\circ} \mathrm{C}$ for $12 \mathrm{~h}$. Then was redispersed in ethanol solvent. Drug loading was calculated as follows:

DL\% $=$ (weight of the drug in nanoparticles)/ (weight of the nanoparticles) $\times 100 \%$

Entrapment efficiency (EE\%) of tamoxifen in MCS was calculated by using the following equation:

$\mathrm{EE} \%=($ weight of the drug in nanoparticles)/ (weight of the feeding drug) $\times 100 \%$

The unloaded tamoxifen was determined by measuring the drug's concentration in the supernatant by UV-Vis spectrophotometry at a wavelength of $250 \mathrm{~nm}$.

The release kinetics of formulations were evaluated by zero-order, first-order, Korsmeyer-Peppas model, and Higuchi's kinetic models.

\subsection{Drug release study.}

$10 \mathrm{mg}$ of freeze-dried MCS was placed into a dialysis bag in a flask containing $15 \mathrm{~mL}$ of phosphate-buffered saline solution (PBS, $\mathrm{pH} 7.4$, and $\mathrm{pH}=5.4$ ) with $0.5 \%$ Tween 80 . The flask put in a shaking incubator (SI-1000, Heidolph, Germany), and at predetermined time intervals, $0.5 \mathrm{ml}$ of samples were withdrawn, and $0.5 \mathrm{ml}$ of fresh PBS was replaced to maintain sink conditions. The amount of released drug was monitored by measuring samples' absorbance using UV spectrophotometer at $250 \mathrm{~nm}$ (Thermo Fisher Scientific, GENESYS 10S, Waltham, MA). The cumulative amount and percent of tamoxifen released from the nanoparticles were calculated. All the release studies were carried out in triplicate.

\subsection{Magnetic resonance imaging (MRI).}

MCS as an MRI contrast agent was used to determine the longitudinal $\left(\mathrm{T}_{1}\right)$ and transversal $\left(\mathrm{T}_{2}\right)$ relaxation times and calculating $\mathrm{r}_{1}$ and $\mathrm{r}_{2}$ relaxivities by using a clinical $1.5 \mathrm{~T}$ 
whole-body magnetic resonance (MR) scanner (Siemens Healthcare Avanto Germany) at $25^{\circ} \mathrm{C}$. $\mathrm{T}_{1}$ and $\mathrm{T}_{2}$ weighted phantom MRI images were obtained in a series of a colloidal suspension of nanoparticles with iron concentrations of $0,25,50,75$, and $200 \mu \mathrm{M}$.

The $\mathrm{T}_{1}$ relaxation times were estimated by applying the spin-echo imaging sequence at various repetition times of 100, 1550, 3150, 4750, and $6400 \mathrm{~ms}$ with an echo time of $18 \mathrm{~ms}$, slice thickness:7.5 mm, the field of view (FOV): 230, and matrix: $200 \times 256$.

The $\mathrm{T}_{2}$ relaxation values were determined to apply the spin-echo sequence with repetition times (TR) of $1600 \mathrm{~ms}$ and varying echo time (TE) of 10, 43, 75, 107, and $140 \mathrm{~ms}$, slice thickness: $7.5 \mathrm{~mm}$, the field of view (FOV): 238 , Turbo factor: 18 , matrix: $176 \times 384$.

The $T_{1}$ and $T_{2}$ relaxation values were determined from mean signal intensities within a manually drawn region of interest (ROI) for each sample, measured with the help of DicomWorks 1.3.5 software. The signal intensity vs. TR or TE functions were exponentially fitted based on the following equations [20, 21]:

$$
\begin{aligned}
& I=M_{0}\left[1-\exp \left(-\frac{T R}{T_{1}}\right)\right] \\
& I=M_{0} \exp \left(-\frac{T E}{T_{2}}\right)
\end{aligned}
$$

Where I is the signal intensity, and $\mathrm{M}_{0}$ is constants. Relaxation rate $\mathrm{R}_{1}\left(1 / \mathrm{T}_{1}\right)$ and $\mathrm{R}_{2}\left(1 / \mathrm{T}_{2}\right)$ were calculated using equations 3 and 4 . By plotting $R_{1}$ and $R_{2}$ over Fe concentration of synthesized MNPs, the slope indicates the specific relaxivity, $\mathrm{r}_{1}$, and $\mathrm{r}_{2}$, respectively.

\subsection{Statistical analysis.}

All experiments were done in triplicate, and the results are reported as the mean \pm standard deviation.

\section{Results and Discussion}

This study is about the synthesis of copolymer-coated magnetic nanoparticles used as a tamoxifen delivery system. The nanoparticle structure with a hydrophobic core and hydrophilic outer shell provides a suitable environment for the transport of both hydrophobic and hydrophilic drugs. In a general sense, mPEG-PCL is self-assembled into a nanosphere with a core-shell structure in the presence of MNPs. It is hypothesized that in the presence of oleic acid-coated magnetite nanoparticles in the oil phase, they are located in the molecular core by the interaction between oleic acid and hydrophobic PCL block. In contrast to magnetite's hydrophobic nature, such a phenomenon causes the particles to be dispersible in water owing to mPEG block covered the particle surface and prevents vicinity to neighboring particles.

\subsection{Copolymer characterization.}

mPEG-PCL di-block copolymer was synthesized using the ring-opening polymerization of $\varepsilon$-caprolactone in the presence of $\mathrm{mPEG}$, whose hydroxyl end groups initiate the ring-opening reaction (Fig 1). 


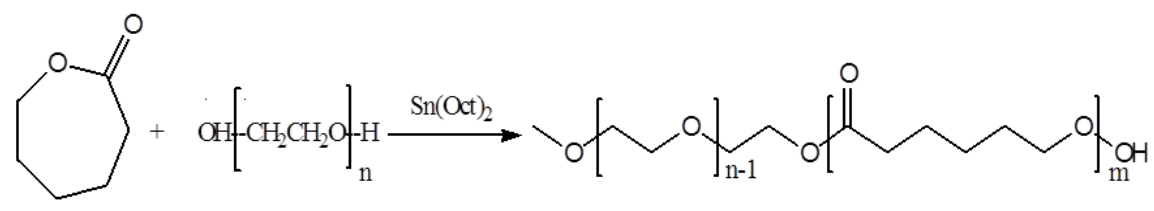

Figure 1. Schematic synthesis route of the mPEG-PCL copolymer.

FTIR spectra (Fig. 2) and ${ }^{1} \mathrm{HNMR}$ (Fig. 3) were utilized to verify the chemical structure of the synthesized mPEG-PCL copolymer. In the FTIR spectrum, the peaks at 2951, 1729, and $1101 \mathrm{~cm}^{-1}$ can be referred to as the alkyl group $(\mathrm{C}-\mathrm{H})$, the ester carbonyl $(\mathrm{C}-\mathrm{O})$, and the ether group (C-O-C) stretching of mPEG-PCL copolymer, one-to-one [22].

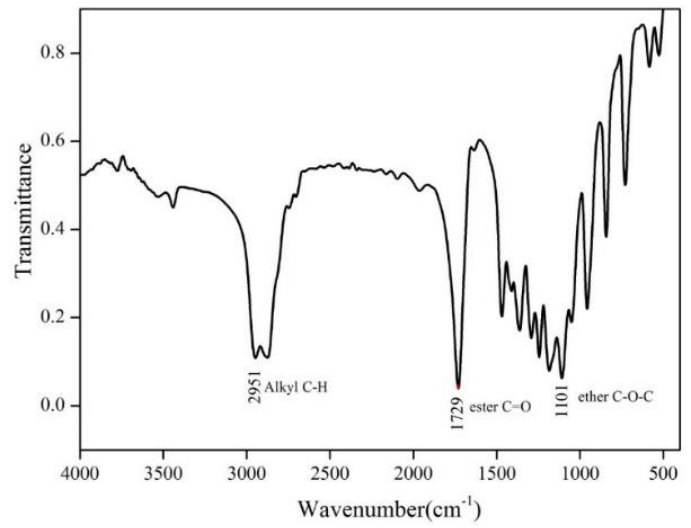

Figure 2. FTIR spectrum of mPEG-PCL copolymer.

In ${ }^{1} \mathrm{HNMR}$ spectrum, the chemical shifts for PEG and PCL protons are as follows: ( $\mathrm{CDCl}_{3}$, ppm, TMS): 3.4 ppm (a, 3H, $\left.\mathrm{CH}_{3}-\mathrm{O}-\right)$, 3.7 ppm (b, 4H, $-\mathrm{O}-\mathrm{CH}_{2}-\mathrm{CH}_{2}-$ ), 4.1 ppm (c, $\left.2 \mathrm{H}, \mathrm{O}-\mathrm{CH}_{2}-\mathrm{CH}_{2}-\mathrm{OCO}\right), 2.3 \mathrm{ppm}\left(\mathrm{d}, 2 \mathrm{H}, \mathrm{CO}-\mathrm{CH}_{2}-\mathrm{CH}_{2}-\mathrm{CH}_{2}-\mathrm{CH}_{2}-\mathrm{CH}_{2}-\mathrm{O}\right), 1.8 \mathrm{ppm}(\mathrm{e}, 4 \mathrm{H}$, $\mathrm{CO}-\mathrm{CH}_{2}-\mathrm{CH}_{2}-\mathrm{CH}_{2}-\mathrm{CH}_{2}-\mathrm{CH}_{2}-\mathrm{O}$ ), $1.4 \mathrm{ppm}$ (f, $2 \mathrm{H}, \mathrm{CO}-\mathrm{CH}_{2}-\mathrm{CH}_{2}-\mathrm{CH}_{2}-\mathrm{CH}_{2}-\mathrm{CH}_{2}-\mathrm{O}$ ), and $4.1 \mathrm{ppm}\left(\mathrm{g}, 2 \mathrm{H}, \mathrm{CO}-\mathrm{CH}_{2}-\mathrm{CH}_{2}-\mathrm{CH}_{2}-\mathrm{CH}_{2}-\mathrm{CH}_{2}-\mathrm{O}\right)$. The HNMR spectrum demonstrates the effective preparation of $\mathrm{mPEG}-\mathrm{PCL}$ copolymer.

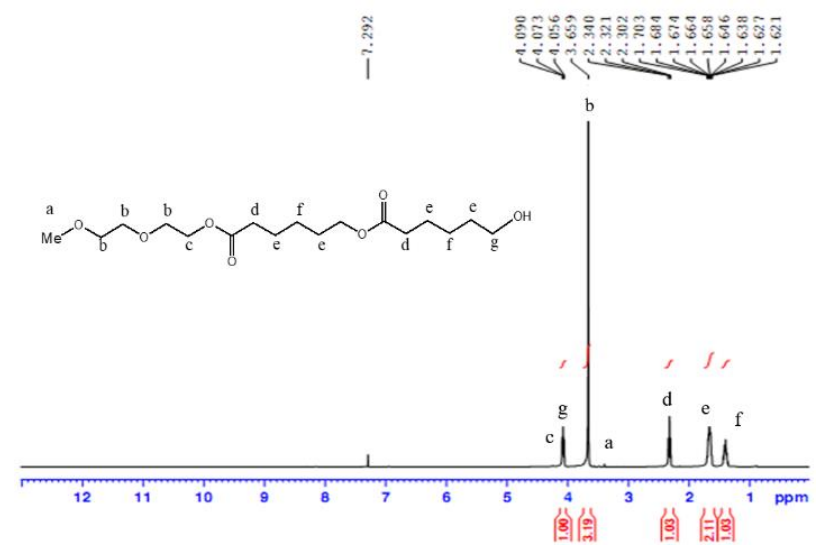

Figure 3. ${ }^{1} \mathrm{H}$ NMR spectrum of the mPEG-PCL di-block copolymer in $\mathrm{CDCl}_{3}$.

PCL's molecular weight in the copolymer backbone was estimated from the integral ratio of the methylene protons adjacent to carbonyl groups in PCL repeating units (peak d, 2.30 $\mathrm{ppm}$ ) methylene proton signals in the repeating units of $\mathrm{mPEG}$. Therefore, the number average molecular weight of synthesized mPEG-PCL block copolymer was calculated to be around 19 KDa.

The melting temperature of the copolymer was measured by DSC (Fig 4). The results revealed that the melting phenomenon of the copolymer is occurring at $54.82{ }^{\circ} \mathrm{C}$. 


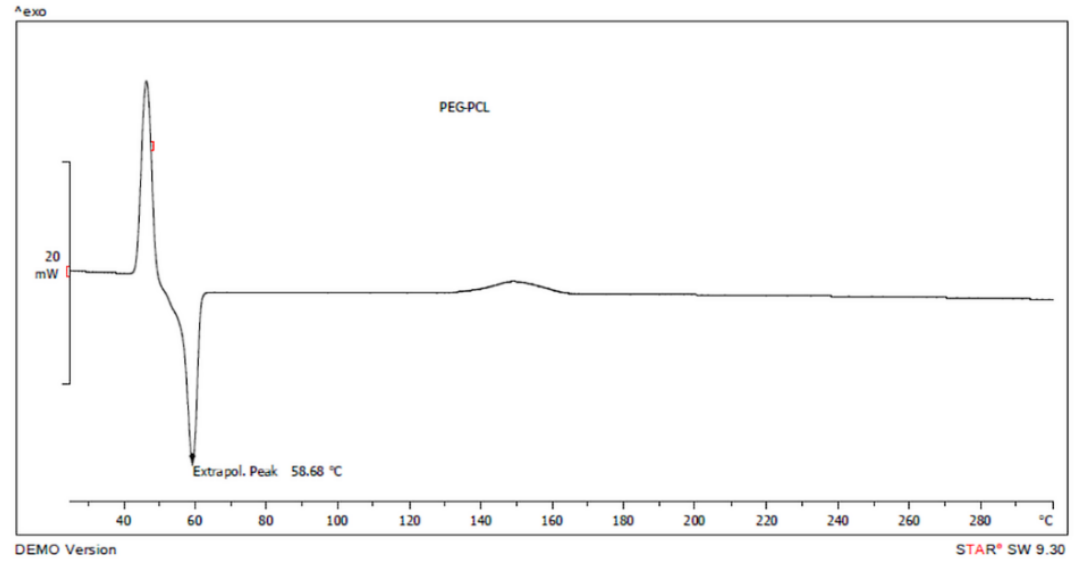

Figure 4. DSC of mPEG-PCL copolymer.

\subsection{Characterization of drug-loaded MCS nanoparticles.}

Fig. 5 shows the FTIR spectra of mPEG-PCL (A), tamoxifen (B), and magnetic mPEGPCL nanoparticles containing tamoxifen (C). In the $\mathrm{C}$ spectrum, the band located at $606 \mathrm{~cm}^{-1}$ in spectrum belongs to $\mathrm{Fe}-\mathrm{O}$ bonds [23], and the band located at $2906 \mathrm{~cm}^{-1}$ can be attributed to the $\mathrm{CH}_{2}$ groups of oleic acid on the surface of magnetite and mPEG. The absorption band at $1728 \mathrm{~cm}^{-1}$ was attributed to the $\mathrm{C}=\mathrm{O}$ stretching vibrations of ester linkages in the PCL block. Tamoxifen as payload drug showed two peaks at 1300 and $1217 \mathrm{~cm}^{-1}$, which are assigned to the $\mathrm{C}-\mathrm{N}$ and $\mathrm{C}=\mathrm{O}$ stretching bands, respectively. All peaks of materials (A) and (B) are visible in the product $(\mathrm{C})$.

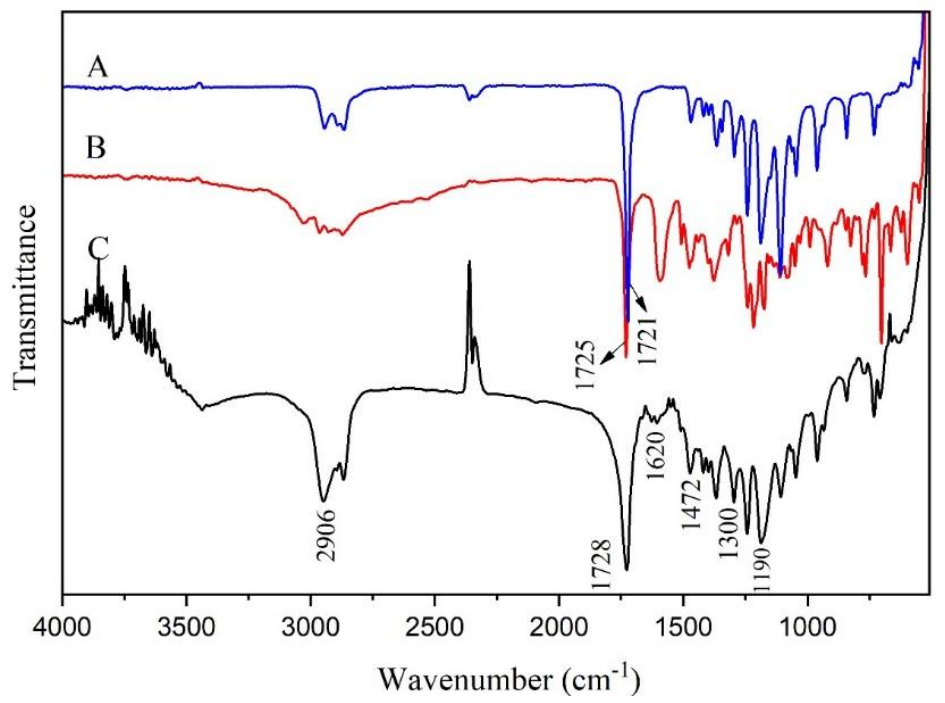

Figure 5. FT-IR analysis of (A) m-PEG-PCL copolymer, (B) tamoxifen and (C) tamoxifen loaded MCS nanoparticles.

\subsection{Particle size, zeta potential, and morphology of MCS nanoparticles.}

The hydrodynamic diameter and the polydispersity index of MCS nanoparticles were done by the DLS instrument. The results were around $183.4 \mathrm{~nm}$ and 0.215 , respectively (Table 1). Considering the particle size of mPEG-PCL prepared with the same procedure, the MCS nanoparticles' hydrodynamic diameters increased due to the presence of the MNPs. The results of the zeta potential analysis of MCS nanoparticles were determined to be around $-5.4 \mathrm{mV}$. The negative zeta potential of nanoparticles may be described by the attendance of some PCL segments on the surface of micelles. 
Table 1. Particular size and zeta potential of tamoxifen loaded and unloaded nanoparticles.

\begin{tabular}{l|c|c|c} 
Sample & Particle size $(\mathbf{n m})$ & Zeta potential $(\mathbf{m V})$ & PDI \\
\hline magnetite & 74.25 & -21.5 & $\mathbf{0 . 3 3 4}$ \\
\hline mPEG-PCL nanoparticles & 120.5 & -5.4 & $\mathbf{0 . 2 1 5}$ \\
\hline Magnetic mPEG-PCL nanoparticles & 183.4 & -4.96 & $\mathbf{0 . 3 9 9}$
\end{tabular}

The SEM analysis was applied to obtain images of the MCS nanoparticles and confirm their figure and morphology. Figure 6 shows the semi-spherical nanostructures with an average diameter of about $80 \mathrm{~nm}$. The aggregation of nanoparticles in SEM image may be due to some magnetic interaction between MCS nanoparticles.

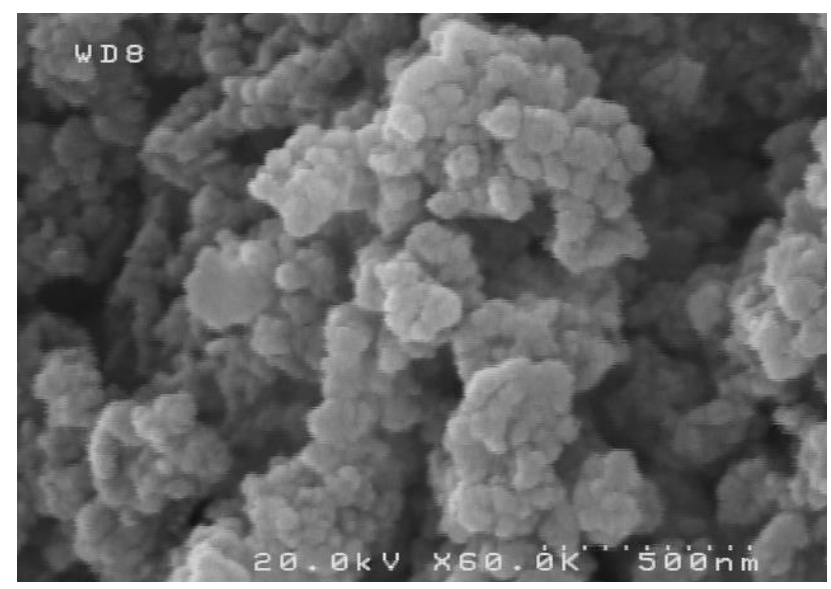

Figure 6. SEM image of MCS nanoparticles.

Fig. 7 shows the TEM image of MCS nanoparticles. It reveals that micelles showed a semi-spherical structure with a mean particle size of around $70 \mathrm{~nm}$.

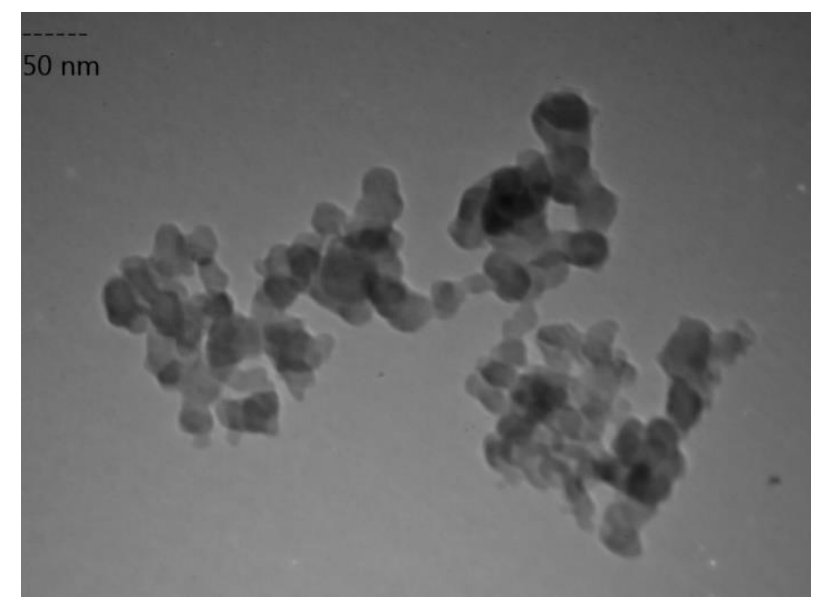

Figure 7. The TEM image of MCS nanoparticles.

\subsection{DSC analysis.}

DSC experiments were tested to determine the physical condition of polymers and drugs in different structures and check for the probability of any interactions amongst drug and nanoparticles. The thermal behavior of the mPEG-PCL copolymer is shown in Fig. 8A. Figure $8 \mathrm{C}$ shows the DSC thermogram of free tamoxifen, a sharp endothermic melting transition at around $143^{\circ} \mathrm{C}$. Figure $8 \mathrm{~B}$ and D are shown the DSC thermograms of tamoxifen loaded MCS nanoparticles and tamoxifen loaded mPEG-PCL micelles (magnetic free nanoparticles) with an endothermic peak at $54.82^{\circ} \mathrm{C}$ and $143{ }^{\circ} \mathrm{C}$, respectively. The appearance of the tamoxifen melting point in the thermogram gives another evidence for encapsulation and the presence of 
the drug in the MCS nanoparticle structure. The slight shift of the melting point of tamoxifen in both mPEG-PCL micelles and MCS nanoparticles compared to the free drug indicates some kind of interaction between the drug and copolymer.

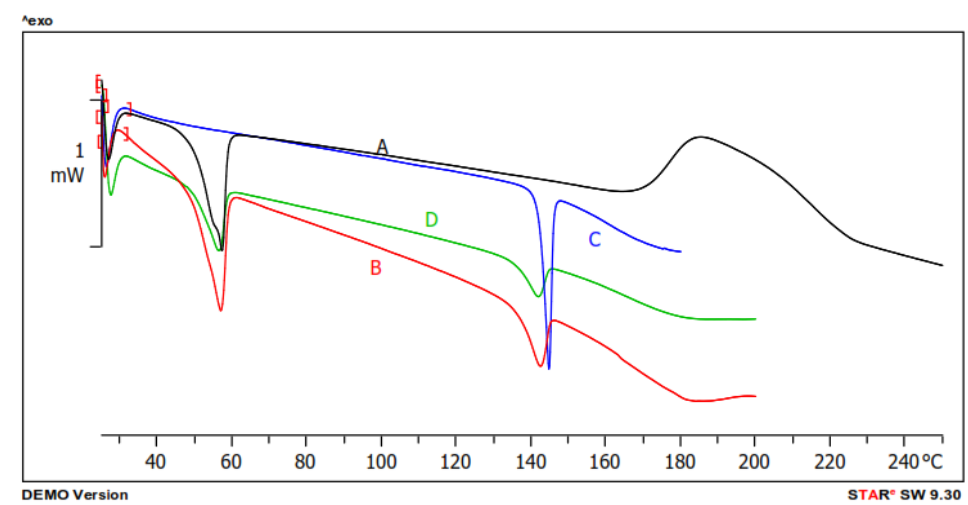

Figure 8. DSC thermograms of (A) mPEG-PCL copolymer, (B) tamoxifen loaded MCS nanoparticles, (C) tamoxifen, (D) tamoxifen loaded mPEG-PCL micelles (magnetic free nanoparticles).

\subsection{Physical stability.}

The size of the nanoparticles was evaluated for up to one week. As shown in Table 2, all nanoparticles show a little increase in their size upon storage. This observation can be explained by the attractive force that arises from magnetite inside the MCS nanoparticles.

Table 2. Physical stability of micelles.

\begin{tabular}{l|l|l|l} 
& \multicolumn{3}{|c}{ Particle size $(\mathbf{n m})$} \\
\hline Time & $\mathbf{1}$ day & $\mathbf{5}$ day & $\mathbf{1 0}$ day \\
\hline magnetite & 74.25 & 74.04 & $\mathbf{7 6 . 4 5}$ \\
\hline mPEG-PCL nanoparticles & 120.50 & 123.60 & $\mathbf{1 2 2 . 4 7}$ \\
\hline Magnetic mPEG-PCL nanoparticles & 183.40 & 185.09 & $\mathbf{1 8 5 . 5 6}$
\end{tabular}

\subsection{Magnetic properties of nanoparticles.}

Vibrating sample magnetometer (VSM) analysis was used to study the magnetic properties of MCS nanoparticles. The magnetization hysteresis loops are shown in Figure 9. The saturation magnetization was found to be 80.12 and $11.69 \mathrm{emu} / \mathrm{g}$ for MNPs and MCS nanoparticles, respectively. The reduction in MCS nanoparticles' saturation magnetization is likely due to the presence of mPEG-PCL coating on the magnetic nanoparticles (i.e., a shell formation process). The same hypothesis has been reported for other nanoparticles [24].

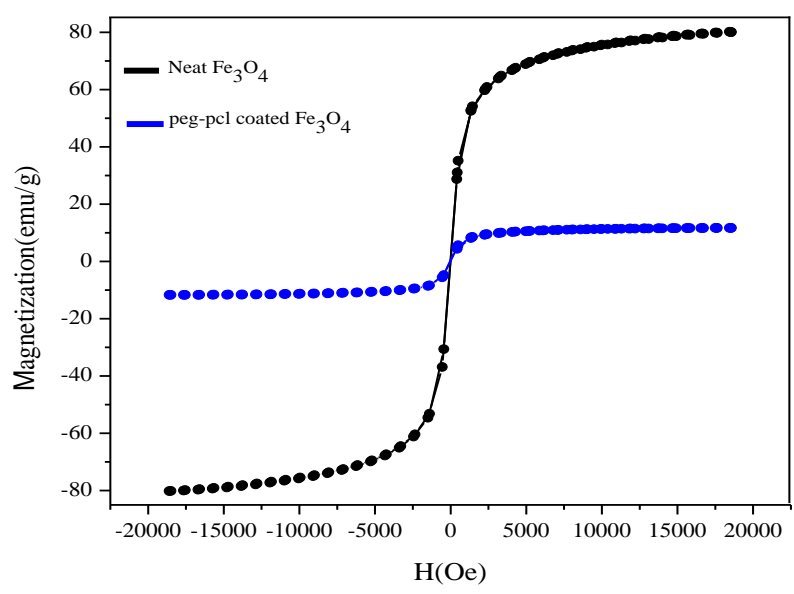

Figure 9. Hysteresis loop by VSM of (a) MNPs, (b) MCS. 


\subsection{Drug-loading parameters.}

The number of encapsulated drugs in the nanoparticles was assayed using UV-Vis spectrophotometer. Entrapment efficiency (\%EE) of the mPEG-PCL micelles (magnetic free nanoparticles) was approximately $70 \%$, and drug loading efficiency (\%DL) was $12 \%$. As clearly shown, tamoxifen has favorable drug-loading efficiency in mPEG-PCL micelles. This desirable value is due to the structural property of the copolymer micelle that entraps the hydrophobic drugs. In the case of MCS nanoparticles, drug loading efficiency and encapsulation efficiency were decreased to $8.14 \%$, and $52.19 \%$, respectively. These results can be due to magnetic nanoparticles' attendance in the core of nanoparticles, limiting drug encapsulation.

\subsection{Drug release study.}

Figure 10 shows the tamoxifen release profiles from MCS nanoparticles. MCS nanoparticles' release behavior was investigated using a dialysis membrane in PBS $(0.5 \%$ Tween 80) at a physiological $\mathrm{pH}$ of 7.4 and an acidic $\mathrm{pH}$ of 5.4 at $37{ }^{\circ} \mathrm{C}$. Given MCS nanoparticles' drug release behavior, it was clear that it exhibited a $\mathrm{pH}$-sensitive release profile, with the higher release in acidic $\mathrm{pH}[25,26]$. The drug release mechanism from MCS nanoparticles relies mainly on a diffusion process from the hydrophobic inner part through the copolymeric segments constituting the nanoparticle. The drug release information in the present study was the best fit for Korsmeyer- Peppas equation. Our experimental data satisfied the linear fitting up to $75 \%$ tamoxifen release from MCS with the $n$ value of $0.68(\mathrm{R} 2=0.9849)$.

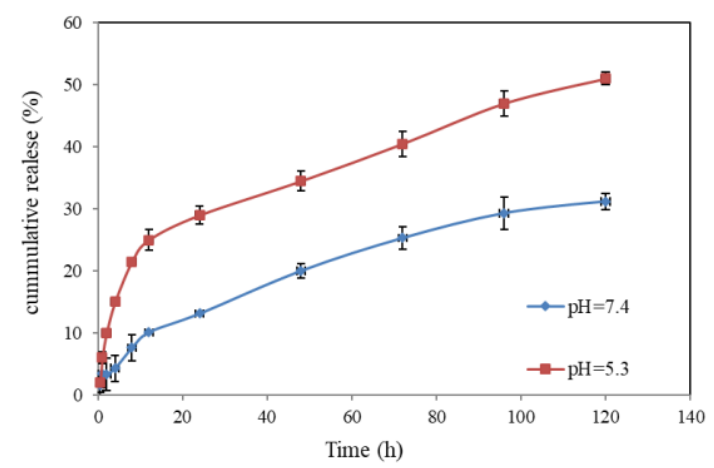

Figure 10. The drug release profile of MCS nanoparticles at the $\mathrm{pH}$ values of 7.4 and 5.3.

\subsection{MRI contrast enhancement.}

The effect of nanoparticles on the longitudinal $\left(\mathrm{T}_{1}\right)$ and transverse $\left(\mathrm{T}_{2}\right)$ proton relaxation was evaluated using a clinical $1.5 \mathrm{~T}$ whole-body magnetic resonance (MR) scanner. Fig. 11 shows $\mathrm{T}_{1}$-weighted MR images of MCS nanoparticles with iron concentrations of 0 , $25,50,75,100$, and $200 \mu \mathrm{M}$ in deionized water. An increase in the phantom images' pixel intensity with the increasing of Fe concentration was clearly observed in the Tiweighed MR images. Longitudinal relaxivity $\left(\mathrm{r}_{1}\right)$ values calculated by the linear fit of the following equation 5:

$$
R_{i}=\frac{1}{T_{i}}=\left(\frac{1}{T_{i}}\right)_{0}+r_{i} C
$$

Where $\mathrm{R}_{\mathrm{i}}$ is the relaxation rate, $\mathrm{T}_{\mathrm{i} 0}$ is the relaxation time in the pure water, $\mathrm{C}$ is the concentration of the contrast agent, and $\mathrm{r}_{i}$ is relaxivity. 


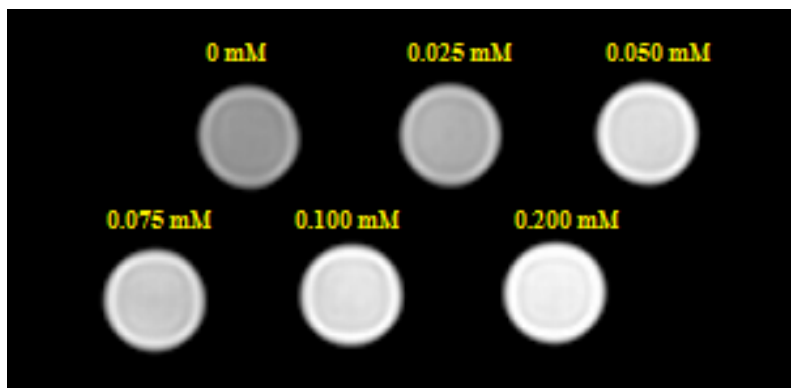

Figure 11. $\mathrm{T}_{1}$-weighted MRI images (1.5T, spin-echo sequence: repetition time $\mathrm{TR}=1550 \mathrm{~ms}$, echo time $\mathrm{TE}=$ $18 \mathrm{~ms}$ ) of the MCS nanoparticles at various iron concentrations at $25^{\circ} \mathrm{C}$.

According to Fig. 12, the longitudinal relaxivity value of $4.171 \mathrm{mM}^{-1} \mathrm{~s}^{-1}$ was observed for MCS nanoparticles. It is clear that the relaxivities are greatly affected by the aqueous medium's distance from the magnetite core. On the other hand, the hydrophobicity/hydrophilicity of the coatings on the surface of magnetic nanoparticles impact water diffusion within the polymeric layer. Thereby, it can be postulated that the presence of hydrophobic inner shells of MCS nanoparticles, including oleic acid and PCL layers, will exclude water molecules and, consequently, extend the water molecules' distance from the magnetite core finally result in low longitudinal relaxivity [27]. It has been reported that magnetite nanoparticles are commonly used as $T_{2}$ MRI contrast agents, and consequently, they are able to decrease the MR signal intensity by dephasing proton spins [28-30]. Figure 12 shows $\mathrm{T}_{2}$-weighted MR images of MCS nanoparticles with iron concentrations.

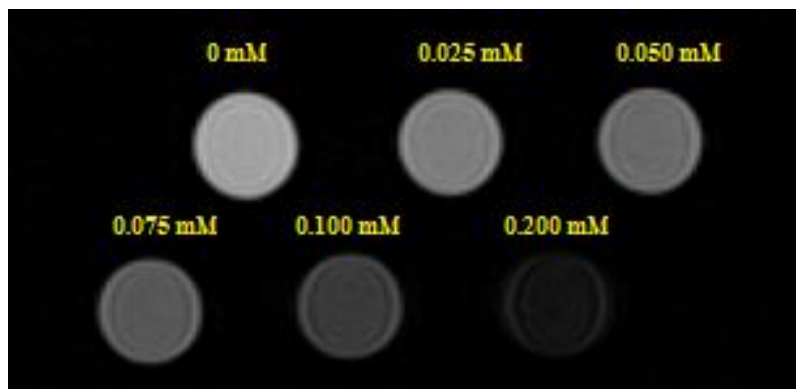

Figure 12. $\mathrm{T}_{2}$-weighted $\mathrm{MRI}$ images $(1.5 \mathrm{~T}$, spin-echo sequence: repetition time $\mathrm{TR}=1600 \mathrm{~ms}$, echo time $\mathrm{TE}=$ $140 \mathrm{~ms}$ ) of the MCS nanoparticles at various iron concentration at $25^{\circ} \mathrm{C}$.

A significant gradual signal drop of the phantom images with increasing concentration of Fe was observed. The $r_{1}$ and $r_{2}$ values calculated from the slope of linear plots of 1/T1 and 1/T2 versus concentration are given in Fig. 13 and 14.

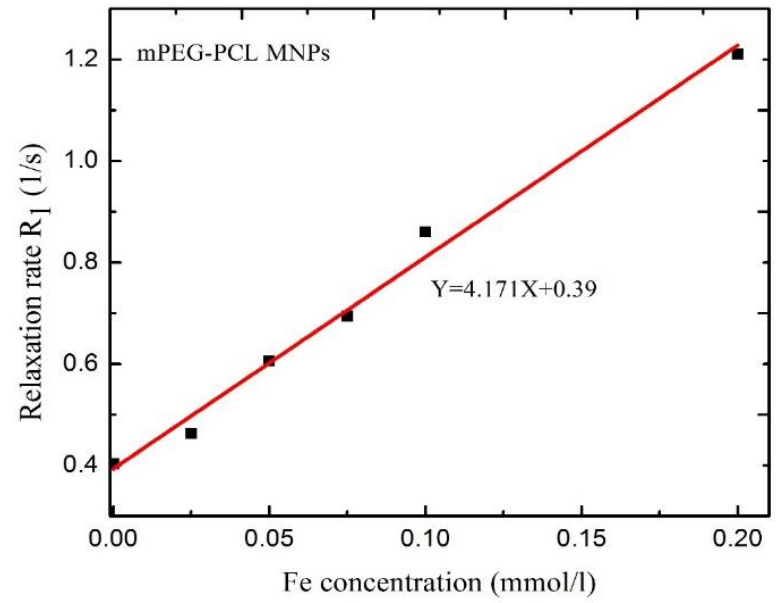

Figure 13. $\mathrm{T}_{1}$ relaxation rate plotted as a function of Fe concentration (mM) for MCS nanoparticles. 


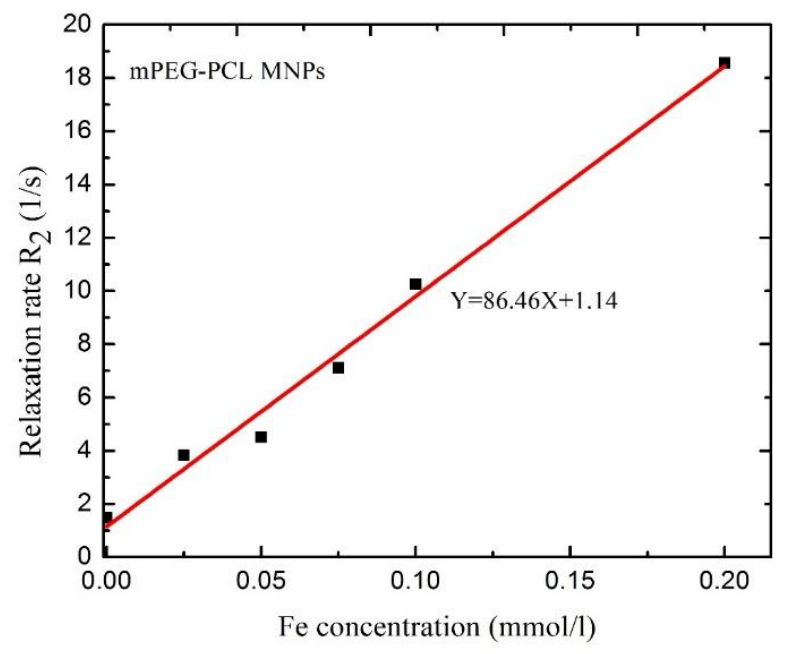

Figure 14. $\mathrm{T}_{2}$ relaxation rate plotted as a function of Fe concentration (mM) for MCS nanoparticles.

A contrast agent's efficiency is determined by the ratio between transverse and longitudinal relaxivity $\left(\mathrm{r}_{2} / \mathrm{r}_{1}\right)$. The $\mathrm{r}_{2} / \mathrm{r}_{1}$ value of as-prepared nanoparticles was around 20 (Table 3), which is higher than that of Reservist, a commercially available MRI contrast agent [31]. This study has hinted at the potential of MCS nanoparticles as T2 MRI contrast agents.

Table 3. The longitudinal relaxivity (r1, mM"1s"1), transverse relaxivity (r2, mM"1s"1), r2/r1values and R2 of chitosan-coated magnetite nanoparticles was calculated by plotting the T1 relaxation rate (1/T1) and T2 relaxation rate $(1 / \mathrm{T} 2)$ as a function of $\mathrm{Fe}$ concentration.

\begin{tabular}{l|c|c|c|c|c} 
Nanoparticles & $\mathbf{r}_{\mathbf{1}}\left(\mathbf{m M}^{-\mathbf{1}} \mathbf{s}^{\mathbf{1}}\right)$ & $\mathbf{R}^{\mathbf{2}}$ & $\mathbf{r}_{\mathbf{2}}\left(\mathbf{m M}^{-1} \mathbf{s}^{-1}\right)$ & $\mathbf{R}^{\mathbf{2}}$ & $\mathbf{r}_{2} / \mathbf{r}_{\mathbf{1}}$ \\
\hline MCS nanoparticles & 4.174 & 0.990 & 86.46 & 0.990 & 20.713
\end{tabular}

\section{Conclusions}

In conclusion, magnetite was synthesized via the co-precipitation method with oleic acid as a stabilizer. After identifying the synthesized compound by different methods, the synthesized mPEG-PCL copolymer was exposed as a shell around the magnetic core. To identify the final magnetic micelles' composition, FT-IR, DLS, TEM, SEM, and VMS spectra were used. Magnetic micelle as a dual agent and drug delivery system was prepared. The nanoparticles drug loading and encapsulation efficiency were $8.14 \%$ and $52.19 \%$, respectively. MCS nanoparticles showed sustained release behavior for $120 \mathrm{~h}$ in the phosphate-buffered saline in $5.3 \mathrm{pH}$ at $37^{\circ} \mathrm{C}$ and were used as a contrast agent for MRI diagnosis to enhance image contrast. It can be concluded that these synthesized nanoparticles have the potential for biomedical applications.

\section{Funding}

This research received no external funding.

\section{Acknowledgments}

The authors are grateful to the Zanjan University of Medical Sciences.

\section{Conflicts of Interest}

The authors declare no conflict of interest. 


\section{References}

1. $\quad$ Long, N.V.; Yang, Y.; Teranishi, T.; Thi, C.M.; Cao, Y.; Nogami, M. Biomedical applications of advanced multifunctional magnetic nanoparticles. Journal of nanoscience and nanotechnology 2015, 15, https://doi.org/10.1166/jnn.2015.11691.

2. Ángeles-Pascual, A.; Piñón-Hernández, J.; Estevez-González, M.; Pal, U.; Velumani, S.; Pérez, R.; Esparza, R. Structure, magnetic and cytotoxic behaviour of solvothermally grown Fe3O4@ Au core-shell nanoparticles. Materials Characterization 2018, 142, https://doi.org/10.1016/j.matchar.2018.05.041.

3. Bekaroğlu, M. G.; Alemdar, A.; İşçi, S. Comparison of ionic polymers in the targeted drug delivery applications as the coating materials on the Fe3O4 nanoparticles. Materials Science and Engineering: $C$ 2019, 103, https://doi.org/10.1016/j.msec.2019.109838.

4. Sirivat, A.; Paradee, N. Facile synthesis of gelatin-coated Fe3O4 nanoparticle: effect of pH in single-step co-precipitation for cancer drug loading. Materials \& Design 2019, 181, https://doi.org/10.1016/j.matdes.2019.107942.

5. Li, D.; Deng, M.; Yu, Z.; Liu, W.; Zhou, G.; Li, W.; Wang, X.; Yang, D.-P.; Zhang, W. Biocompatible and stable GO-coated $\mathrm{Fe} 3 \mathrm{O} 4$ nanocomposite: A robust drug delivery carrier for simultaneous tumor MR imaging and targeted therapy. ACS Biomaterials Science \& Engineering 2018, 4, https://doi.org/10.1021/acsbiomaterials.8b00029.

6. Taghipour-Sabzevar, V.; Sharifi, T.; Moghaddam, M.M. Polymeric nanoparticles as carrier for targeted and controlled delivery of anticancer agents. Therapeutic delivery 2019, 10, https://doi.org/10.4155/tde-20190044.

7. Sung, Y.K.; Kim, S.W. Recent advances in polymeric drug delivery systems. Biomaterials Research 2020, 24, https://doi.org/10.1186/s40824-020-00190-7.

8. Fortuni, B.; Inose, T.; Ricci, M.; Fujita, Y.; Van Zundert, I.; Masuhara, A.; Fron, E.; Mizuno, H.; Latterini, L.; Rocha, S. Polymeric engineering of nanoparticles for highly efficient multifunctional drug delivery systems. Scientific reports 2019, 9, https://doi.org/10.1038/s41598-019-39107-3.

9. Xie, S.; Zhang, B.; Wang, L.; Wang, J.; Li, X.; Yang, G.; Gao, F. Superparamagnetic iron oxide nanoparticles coated with different polymers and their MRI contrast effects in the mouse brains. Applied Surface Science 2015, 326, https://doi.org/10.1016/j.apsusc.2014.11.099.

10. Yoon, J.; Cho, S. H.; Seong, H. Multifunctional ultrasmall superparamagnetic iron oxide nanoparticles as a theranostic agent. Colloids and Surfaces A: Physicochemical and Engineering Aspects 2017, 520, https://doi.org/10.1016/j.colsurfa.2017.02.080.

11. Piazza, R.D.; Brandt, J.V.; Gobo, G.G.; Tedesco, A.C.; Primo, F.L.; Marques, R.F.C.; Junior, M.J. mPEGco-PCL nanoparticles: The influence of hydrophobic segment on methotrexate drug delivery. Colloids and Surfaces A: Physicochemical and Engineering Aspects 2018, 555, https://doi.org/10.1016/j.colsurfa.2018.06.076.

12. Wei, W.; Li, S.; Xu, H.; Zhou, F.; Wen, Y.; Song, Z.; Feng, S.; Feng, R. MPEG-PCL Copolymeric Micelles for Encapsulation of Azithromycin. AAPS PharmSciTech 2018, 19, https://doi.org/10.1208/s12249-0181009-0.

13. Wang, C.; Qi, P.; Lu, Y.; Liu, L.; Zhang, Y.; Sheng, Q.; Wang, T.; Zhang, M.; Wang, R.; Song, S. Bicomponent polymeric micelles for $\mathrm{pH}$-controlled delivery of doxorubicin. Drug delivery 2020, 27 , https://doi.org/10.1080/10717544.2020.1726526.

14. Day, C.M.; Hickey, S.M.; Song, Y.; Plush, S.E.; Garg, S. Novel tamoxifen nanoformulations for improving breast cancer treatment: Old wine in new bottles. Molecules 2020, 25, https://doi.org/10.3390/molecules25051182.

15. Nankali, E.; Shaabanzadeh, M.; Torbati, M.B. Fluorescent tamoxifen-encapsulated nanocapsules functionalized with folic acid for enhanced drug delivery toward breast cancer cell line MCF-7 and cancer cell imaging. Naunyn Schmiedebergs Arch Pharmacol 2020, 393, 1211-1219, https://doi.org/10.3390/molecules25051182.

16. Zarrin, A.; Sadighian, S.; Rostamizadeh, K.; Firuzi, O.; Hamidi, M.; Mohammadi-Samani, S.; Miri, R. Design, preparation, and in vitro characterization of a trimodally-targeted nanomagnetic onco-theranostic system for cancer diagnosis and therapy. International Journal of Pharmaceutics 2016, 500, https://doi.org/10.1016/j.ijpharm.2015.12.051.

17. Karami, Z.; Sadighian, S.; Rostamizadeh, K.; Hosseini, S. H.; Rezaee, S.; Hamidi, M. Magnetic brain targeting of naproxen-loaded polymeric micelles: pharmacokinetics and biodistribution study. Materials Science and Engineering: C 2019, 100, https://doi.org/10.1016/j.msec.2019.03.004.

18. Sadighian, S.; Rostamizadeh, K.; Hosseini, M.-J.; Hamidi, M.; Hosseini-Monfared, H. Magnetic nanogels as dual triggered anticancer drug delivery: toxicity evaluation on isolated rat liver mitochondria. Toxicology Letters 2017, 278, https://doi.org/10.1016/j.toxlet.2017.06.004.

19. Rostamizadeh, K.; Rezaei, S.; Abdouss, M.; Sadighian, S.; Arish, S. A hybrid modeling approach for optimization of PMAA-chitosan-PEG nanoparticles for oral insulin delivery. RSC advances 2015, 5, https://doi.org/10.1039/C5RA07082A. 
20. Kermanian, M.; Naghibi, M.; Sadighian, S. One-pot hydrothermal synthesis of a magnetic hydroxyapatite nanocomposite for MR imaging and $\mathrm{pH}-$ Sensitive drug delivery applications. Heliyon 2020, 6, https://doi.org/10.1016/j.heliyon.2020.e04928.

21. Lee, N.; Hyeon, T. Designed synthesis of uniformly sized iron oxide nanoparticles for efficient magnetic resonance imaging contrast agents. Chemical Society Reviews 2012, 41, https://doi.org/10.1039/C1CS15248C.

22. Karami, Z.; Sadighian, S.; Rostamizadeh, K.; Parsa, M.; Rezaee, S. Naproxen conjugated mPEG-PCL micelles for dual triggered drug delivery. Materials science and engineering: C 2016, 61, https://doi.org/10.1016/j.msec.2015.12.067.

23. Sadighian, S.; Abbasi, M.; Arjmandi, S.; Karami, H. Dye removal from water by zinc ferrite-graphene oxide nanocomposite. Progress in Color, Colorants and Coatings 2018, 11, https://doi.org/10.30509/pccc.2018.75743.

24. Khalkhali, M.; Rostamizadeh, K.; Sadighian, S.; Khoeini, F.; Naghibi, M.; Hamidi, M. The impact of polymer coatings on magnetite nanoparticles performance as MRI contrast agents: a comparative study. DARU Journal of Pharmaceutical Sciences 2015, 23, https://doi.org/10.1186/s40199-015-0124-7

25. Rashidzadeh, H.; Salimi, M.; Sadighian, S.; Rostamizadeh, K.; Ramazani, A., In vivo Antiplasmodial Activity of Curcumin-Loaded Nanostructured Lipid Carriers. Current Drug Delivery 2019, 16, 923-930, https://doi.org/10.2174/1567201816666191029121036.

26. Ramazani, A.; Abrvash, M.; Sadighian, S.; Rostamizadeh, K.; Fathi, M. Preparation and characterization of curcumin loaded gold/graphene oxide nanocomposite for potential breast cancer therapy. Research on Chemical Intermediates 2018, 44, 7891-7904, https://doi.org/10.1007/s11164-018-3593-8(0123456789().,volV)(0123456789().,-volV).

27. Illés, E.; Szekeres, M.; Kupcsik, E.; Tóth, I.Y.; Farkas, K.; Jedlovszky-Hajdú, A.; Tombácz, E. PEGylation of surfacted magnetite core-shell nanoparticles for biomedical application. Colloids and Surfaces A: Physicochemical and Engineering Aspects 2014, 460, https://doi.org/10.1016/j.colsurfa.2014.01.043.

28. Naghizadeh, M.; Taher, M.A.; Tamaddon, A.-M.; Borandeh, S.; Abolmaali, S.S. Microextraction of Gadolinium MRI contrast agent using core-shell Fe3O4@ SiO2 nanoparticles: optimization of adsorption conditions and in-vitro study. Environmental Nanotechnology, Monitoring \& Management 2019, 12, https://doi.org/10.1016/j.enmm.2019.100250.

29. Kang, N.; Xu, D.; Han, Y.; Lv, X.; Chen, Z.; Zhou, T.; Ren, L.; Zhou, X. Magnetic targeting core/shell $\mathrm{Fe} 3 \mathrm{O} 4 / \mathrm{Au}$ nanoparticles for magnetic resonance/photoacoustic dual-modal imaging. Materials Science and Engineering: C 2019, 98, 545-549, https://doi.org/10.1016/j.msec.2019.01.013.

30. Liu, D.; Li, J.; Wang, C.; An, L.; Lin, J.; Tian, Q.; Yang, S. Ultrasmall Fe@ Fe3O4 nanoparticles as T1T2 dual-mode MRI contrast agents for targeted tumor imaging. Nanomedicine: Nanotechnology, Biology and Medicine 2020, 32, https://doi.org/10.1016/j.nano.2020.102335.

31. Ma, X.; Gong, A.; Chen, B.; Zheng, J.; Chen, T.; Shen, Z.; Wu, A. Exploring a new SPION-based MRI contrast agent with excellent water-dispersibility, high specificity to cancer cells and strong MR imaging efficacy. Colloids and surfaces. B, Biointerfaces 2015, 126, https://doi.org/10.1016/j.colsurfb.2014.11.045. 